\title{
Machine Learning based Methodology Process Model to Handle Healthcare Information
}

\author{
K. Subrahmanyam, G. Bala Bharath Reddy, T.V.L. Manisha, K. Raga Poojitha, B. Yashaswi \\ Department of Computer Science and Engineering, K L University, Vaddeswaram, Guntur, A.P, India \\ *Corresponding author: E-Mail: gorlabalabharathreddy95@gmail.com
}

\section{ABSTRACT}

The Machine Learning (ML) areas include obtained its strength in about anyone sector from scholar too deserved newly had grown a dependable device into their medical care sector. Their scientific sector from electronic teaching is used to charge such as medical care conclusion assist, medical picture, protein-protein connections, removal from medical care understanding, too from overall individual administration proper management. Machine Learning is visualize as a device by which computer-based systems can be incorporated into their medical care area in order to get a better, more well-organized medical proper care. This Document provides efficient device learning algorithms and methods used in getting sickness and treatment related phrases from brief written text released in medical documents. Primary employment to display what Natural Language Process Handling (NLP) and Machine Learning methods used for reflection of information and what category methods are appropriate for identifying \& identifying appropriate medical care details in brief written text. This paper also present medical care analysis therapy \& prevention of sickness, injury in human. The sector is automatically learned some task of medical care details, medical control, and individual health details etc. The used mechanism can be incorporated with any medical management program to make better medical care decision for a human control program can instantly use mining techniques to get biomedical details from digital databases.

KEY WORDS: Healthcare, Machine Learning, Natural Language Processing, Support Vector Machine, Decision Support System.

\section{INTRODUCTION}

People care greatly about their wellness and want to be, now more than ever, in cost of their wellness and health. Life is more stressful than ever, the medication that is practiced nowadays is an Evidence Based Medicine (hereafter, EBM) in which healthcare skills is not only depending on years of practice but on the newest findings as well. Resources that can help us handle and better keep a record of our wellness such as Google Health insurance coverage, Microsoft Company Health-Vault are factors and facts that get individuals more highly effective when it comes to healthcare knowledge and control. The conventional medical care program is also becoming one that holds the online and the digital globe. This Platform provides the base for growth of technology structure that makes readily available all the relevant details regarding therapy and illnesses. The tool that is designed with the methods such as Natural Language Process Handling (NLP) and Machine Learning (ML) has ability to discover all appropriate brief written text information regarding illnesses and therapies. The work presents various Machine Learning (ML) techniques and details for classifying brief written texts and regards between illnesses and treatments.

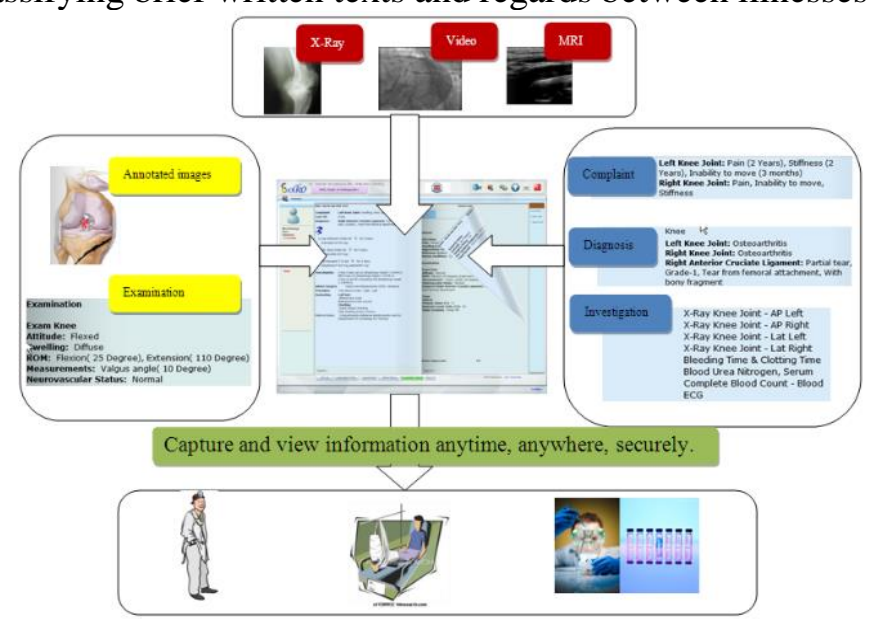

Figure.1. Automatic medical case sheet classification for fast understanding of patient's profile

According to Machine Learning strategies the details are shown in brief written texts when determining interaction between two organizations such as illnesses and therapy. Thus there is improvement in alternatives when using a direction of two tasks (Hierarchical way of approaching). While exists superior though recognize too take their phrase while does not accommodate data appropriate to illness or therapies. The remaining phrases can be categorized according to the interest. It will be very complicated to recognize the exact solution if everything is done in one phase by classifying sentences depending on attention and also such as the sentences that do not offer 


\section{Sun International Journal of Engineering and Basic Sciences}

appropriate details. Relation Extraction is a traditional analysis subject in Natural Language Handling. Healthcare details are saved in textual structure among the scientific information saved in Medline. Generally getting helpful details were big sound from information source to boring work. Even more showing scientific details contain medical data too generally irrelevant components even more navigation choices, types, customer feedback, marketing, feedback etc. The suggested work of these venture ingredients the useful illness relevant details with improved processing being using calculated bag from term reflection which an accuracy from $79 \%$ to $82 \%$. Their suggested strategy helps in medical handling decisions being offering doctor with better available proof from medical details. In this paper we choose text exploration focused with medical papers relevant through Medical therapy. Medline being selected into venture through get biomedical details being it proves solutions relevant to individual therapy and it's the data source which is most widely used by the physicians too analysis students into medical area. Even important it is mostly updated too their material are turned through be precise in comparison to healthcare sites offering details relevant to human illness, health, medications, therapy etc. With the growing number of healthcare dissertation, analysis documents, research articles, scientists have to face the problems from studying a lot to analysis documents to obtain skill in their area of interest. Google like Pub Med decreases this restriction by accessing the appropriate papers appropriate to the customer question. Here venture were their irrelevant material like marketing etc. described into the above passage are eliminated too text exploration is conducted onto their extracted paper to details or phrases appropriate through customer mentioned illness being produced. Through their produced file symptoms, causes, treatment from their particular illness is strained and shown to the customer. Thus their customer gets the required details alone which helps you to save his time and enhances their quality from result. In the suggested approach a mixture of Architectural Natural Language handling with machine studying methods to address the general and sector specific difficulties of details removal. Healthcare subheadings and subject going may be used to infer relationship among medical ideas. The category criteria used in the suggested work displays performance, performance, and online studying ability.

\section{METHODS \& MATERIALS}

Background Work: The most appropriate relevant perform is to perform done by Rosario and Hearst. Details places were used for obtaining our program successfully. The data set includes phrases too Medline abstracts interpret with illness too presentation organizations and with eight semantic interaction linking illnesses too therapies. Their primary objective from performance is to enterprise identification from illness too therapies. In biomedical literary works, rule-based techniques exist commonly pre-owned from fixing regards removal charge. Their primary resources worn being these strategies exist either syntactic: part-of-speech (POS) too syntactic structures; or semantic data by means from set styles that accommodate terms that induce a certain interpret. One of the disadvantages from utilize techniques depending onto guidelines is that they usually demand further more human-expert attempt than data-driven techniques (though individual attempt is required in data-driven techniques too, to brand the data). The best rule-based techniques are the ones that use guidelines designed personally or semi-automatically withdraw instantly too enhanced personally. A beneficial part from rule-based techniques is the fact that they acquire fine perfection consequence, interval their remember stages are generally low. Syntactic rule-based regards removal techniques are complicated techniques depending onto additional resources used to allocate POS labels or to draw out syntactic parse plants. It is known that in the biomedical literary works such resources are not yet at the state of the art level as they are for common British written texts, and therefore their efficiency on phrases is not always the best. Associate works on syntactic rule-based techniques for regards removal in Medline abstracts and full-text content are provided by Johnson, Yakushiji, and Leroy. Even though the syntactic details are caused by resources that are not 100 percent precise, testimonials with these types of techniques have been experienced in the biomedical sector. To do this work effectively traditionally perform two tasks for downloaded data sets. The two tasks that are performed in conventional machine learning work provide the foundation for the design of the technology framework that is capable to recognize and spread medical care details. The first process recognizes and ingredients useful phrases on illnesses and therapies subjects, while the second one works a better grained category of these phrases according to the semantic interaction that prevails between illnesses and therapies. The first process (task 1 or phrase selection) recognizes phrases from Medline released abstracts that discuss illnesses and therapies. The process is just like a check out of phrases in the subjective of a content to be able to existing to the user-only phrases that are recognized as containing appropriate details (disease treatment information). The second process has a further semantic sizing and it is targeted on determining disease-treatment interaction in the phrases already chosen as being useful. We concentrate on three relations: Cure, Prevention, and Side effects, a part of the eight interactions that the corpus is annotated with. We made the decision to pay attention to these three interactions because these are most showed in the corpus while for the other five, very few illustrations are available.

Proposed Approach: The two projects used in this paper are the basis for the development of technology structure. This structure allows recognizing the healthcare relevant details from abstracts. The first process offers with removal 


\section{Sun International Journal of Engineering and Basic Sciences}

all details regarding illnesses and therapies while the task offers with removal of relevant details existing between illness and therapies. The structure designed with these projects are used by healthcare care providers, individuals who needs to manage their healthcare relevant issues and organizations that build systematic views. The future item can be offered with browser plug-in and desktop application so that it allows the customer to get all details relevant to illnesses and therapies and also the regards between those entities. It is also be useful to know more about latest findings relevant to medicine. The item can be designed and sold by organizations that do analysis in wellness proper care domain using Natural Language Process Handling (NLP), and Machine Learning (ML), and organizations that create resources like Microsoft Health Container and Google Health. This item is valuable in e-commerce areas by showing the statistics that the details offered here are precise and also offer all the latest findings relevant to wellness proper care. To make an item more popular it should be believe in deserving so that individuals can buy it. It is the key factor for any company to make item successful. When coming to wellness maintenance systems it should be more believe in deserving since it is dealing with healthcare relevant issues. Businesses that wish to sell wellness proper care structure need to create resources that automatically draw out the wealth of analysis.

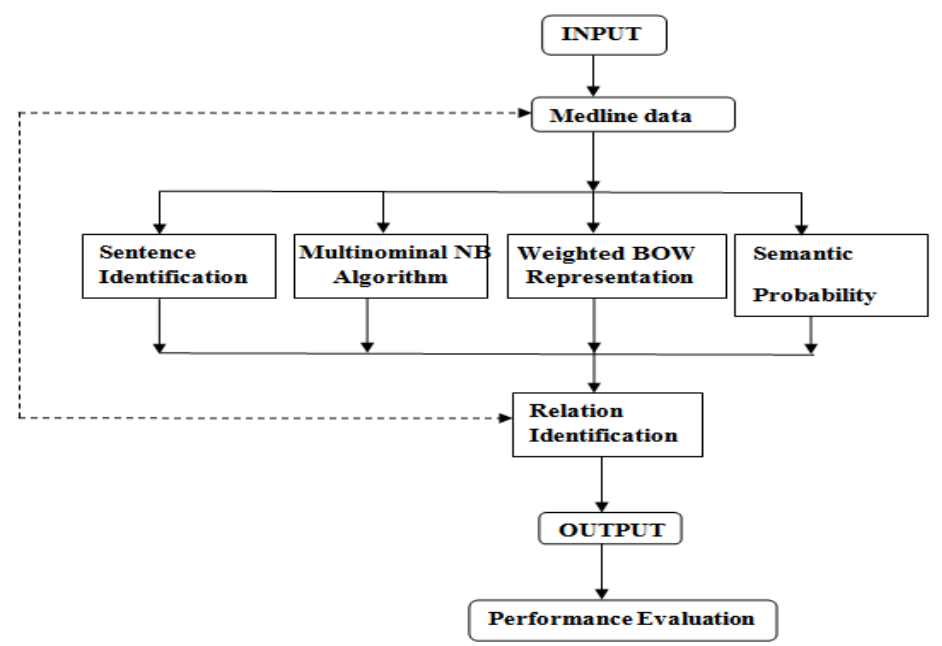

Figure.2. Proposed architecture for solving data sets based on their relevancy of key word search

For example the details offered for illnesses or therapies needs to be based on latest findings on wellness proper care area so that individuals can believe in. The high quality also should be taken proper care so that it provides dynamic content for users. The first process offers with the identification of phrases from the Medline abstracts that offer the information about the illnesses and therapies. In other words it also seems like scanning the phrases from Medline abstracts that contain relevant details which the customer wants. Natural Language Process Handling (NLP), and Machine Learning (ML) are used to draw out precise details or it can also say that it perfectly removes the unwanted details which are not relevant to illness or therapy. Natural Language Process Handling (NLP) and Machine Learning (ML) itself involve in getting useful phrases. It is trial to recognize the informative phrases in areas such as summarization and details removal. The work and contribution value with this process is helpful in results and in settings for this process in healthcare care area. Being semantic regards to details relevant through Symptoms, Causes too Treatments for certain illness in the customer submitted html file. In order to draw out their semantic relations a category criteria namely Multinomial Naïve Bayes category criteria is used in functioning with a prior organization rule mining. Their reason for choosing Multinomial Naïve Bayes and the drawback of Naïve Bayes criteria are discussed below. Multinomial Naïve Bayes is the specialized version of Naïve Bayes specially used for text papers. Multinomial Naïve Bayes models the word count and performs the category within it. Word series design strategy is used to evaluate information to recognize the design; such styles can be used to make forecast which is an effecting method in making decisions. It is used to recognize design to medical care sector to find design seen in the symptoms of different illness.

\section{Performance Evaluation:}

Data Sets Extraction: In section 2 and section 3 we define two tasks, the two projects that are performed in this document offer the basis for the style of a details technology framework that is able to recognize and disseminate healthcare details. The first process recognizes and extracts informative phrases on illnesses and therapies subjects, while the second one works a better grained classification of these phrases according to the semantic interaction that exists between illnesses and therapies.

Bag of Words: Here, Bag of Words (BOW) reflection being generally using for written test category projects. Been a reflection while features are selected between their terms that were existing into teaching information. Choice methods being to be able find their more appropriate terms being functions. After their present area is recognized, each coaching too analyse example is mapped to this function reflection being providing principles to every function 


\section{Sun International Journal of Engineering and Basic Sciences}

from different example. Two most common feature value representations for BOW reflection are: binary function values, the value of a function can be either 0 or 1 , where 1 symbolizes the point that the function is present in the example and 0 otherwise; or regularity feature values - the value of the function is how frequently it appears in an example, or 0 if it did not appear. Being we deal with brief written texts with a normal of 20 terms per sentence, their distinction between a binary value presentations to a regularity utility reflection is not large. In our situation, we select a regularity value representation. IT has been benefits that if a feature appears more than once in a phrase, this implies that it are important and their regularity valuation reflection will be reproduce this.

Genie Tagger: Kind of reflection is based on syntactic information: noun-phrases, verb-phrases, and biomedical ideas recognized in the words. In order to draw out this kind of details, we used the Genia11tagger device. The tagger examines the words and results the base types, part-of-speech labels, amount labels, and known as enterprise labels. The tagger is specifically updated for biomedical written text such as Medline abstracts. The noun too verb-phrases recognized being their tagger were functions used for their second reflection strategy. We ran their Genie tagger onto their whole information set.

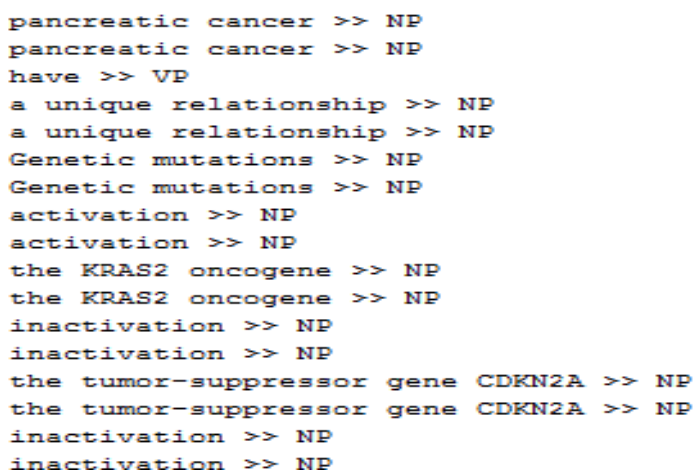

Figure.3. Practical application development in pub med data extraction

For this present process, determining which words from the abstracts of Medline content that contain useful words for illnesses and therapies, the best outcomes acquired. The reflection strategy that uses BOW functions, UMLS ideas, noun and verb-phrases, and biomedical ideas with the CNB classifier acquire a $90.72 \%$ F-measure and $90.36 \%$ precision. These improves in outcomes are due to the point that all these various kinds of functions make a wealthy and predictive feature area for the classifiers. The concentrate for the second process is to instantly recognize which phrases contain details for the three semantic relations: Treat, Avoid, and Part Impact. The revealed outcomes are depending on identical configurations to the ones used for the past process. Since unbalanced details places are used for this process, the assessment evaluate that we are going to review is the F-measure. Due to area problems, we are going to present the best outcomes acquired for all configurations. The best outcomes are selected from all the reflection methods and all category methods that we also used for the first process. The NB classifier mixtures from different reflection functions is the one that acquires the best outcomes for all interaction. Their enhancement over the other configurations can be due to their point that the mixture of classifier and functions has an excellent forecasting worth from design qualified on the three interaction.

\section{EXPERIMENTAL RESULTS}

This section talks about the outcomes we acquired for the two tasks in this research. For the first process, the one of identifying informative phrases, the outcomes display that probabilistic models depending on Naive Bayes system, acquire great outcomes. The proven reality that the SVM classifier works well reveals that the present findings are in range with the literary works. These two classifiers have always been proven to execute well on text category projects. Even though the freedom of features is breached when using Naive Bayes classifiers, they still execute very well. In this paper we present to develop efficient and effective data transmission with considering the processing of application development. We calculate individual score of each relation based on expressed biomedical relations of each preferred process on data sets with semantic relations.

Table.1. Data Score of related data items

\begin{tabular}{|l|l|l|}
\hline Abstract Number & SVM Classifier & CNB Classifier \\
\hline 1 & 96 & 77 \\
\hline 2 & 91 & 69 \\
\hline 3 & 98 & 90 \\
\hline 4 & 97 & 52 \\
\hline 5 & 96 & 79 \\
\hline
\end{tabular}

One of the significant efforts of this work is the fact that the current tests display that more details in the reflection configurations delivers developments for the task of determining useful phrases. 


\section{Sun International Journal of Engineering and Basic Sciences}

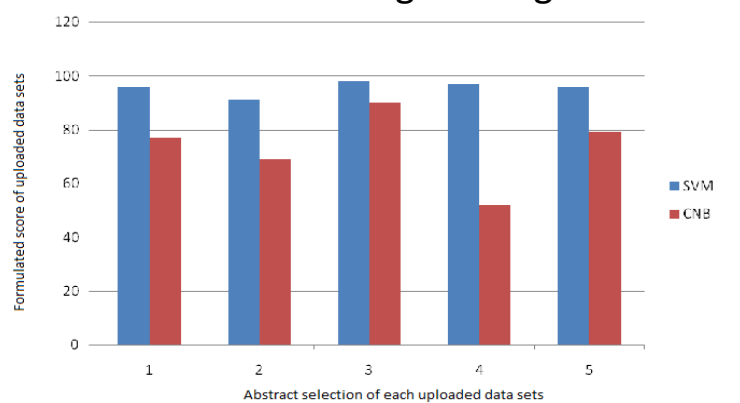

Figure.4. Formulated score of the uploaded data sets

The experimental results show efficient communication of the data sets retrieval with processing of identified data items based on their relevant data processes. The procedure itself is a realization-charged task; the marking procedure includes a human-intensive annotation procedure being interaction different entities needs to be personally recognized. Their enquiry structured from the automated procedure aim to demonstrate that classifiers execute superior while better details are offered. We are also calculating time efficiency of each uploaded data sets. The reflection and the category methods while able through create their difference between the interactions too acquire their best outcomes from these process. Identical findings as their ones acquired from their first process were reasonable: probabilistic models along with more useful function representation bring the best outcomes. As portrayed in the figure, the outcomes acquired in this research outshine the previous ones. In one situation, the same low outcomes are obtained; for example, for the No-Cure category, the low outcomes are due to the proven reality that this category is underrepresented in the information set, by only four illustrations in complete. Their category Unexplained acquires different outcomes when one model is used from all interaction, but it outperforms past solutions when the better design is selected from their category.

\section{CONCLUSION}

Here, suggested system eliminates the undesirable material from the subjective to Medline and outcome on a written text papers carry only their specific illness too appropriate Symptoms, Cause too therapy. Trial outcome reveals that the strategy used in the suggested perform reduces the time and performance of the physicians in examining information about certain illness and treatment to help make decision about individual tracking and treatment. This written text excavated papers can be used in medical care sector where a physician can analyse various kinds of treatment that can be given to individual with particular healthcare problem. the experimental methodology when the first establishing is used for the second process, to use extra resources were representing methods, were to concentrate on methods to combine their analysis findings in a structure to be position from customers. Moreover to more methodological settings in which we try to discover their prospective utility from other types too presentation, we would like through pay attention to source information that comes from their web. Determining too classifying medical-related details on to web is a process that can carry useful details to their analysis similarity too also their end customer.

\section{REFERENCES}

Abdur Rehman, Haroon Babri A, Mehreen Saeed, Feature Extraction Criteria for Category of Written text Document, ICCIT 2012.

Adrian Canedo-Rodriguez, Jung Hyoun Kim, Efficient Text Extraction Algorithm Using Shade Clustering For Language Translation In Cellular Phone, Journal of Signal and Information Processing, 2012.

Jerr Rennie D.M, Lawrence Shih, Jaime Teevan, Bob Karger R, Tackling The POOR Supposition Of Naïve Bayes Text Classifier, Procedures Of The 20th Worldwide Conference On Device Studying (ICML-2003), California DC, 2003.

Mark Pasternack, Don Roth, Extracting Content Written text From Webb with Highest possible Subsequence Segmentation, 2009.

Oana Frunza, A Device Studying Strategy For Identifying Disease-Treatment Interaction In Brief Texts, 2011

Oana Frunza, Diana Inkpen, and Johnson Tran, A Machine Studying Strategy for Determining Disease-Treatment Interaction in Brief Texts, IEEE dealings on knowledge and details technological innovation, 23 (6), 2011.

Rosario B and Hearst M.A, Semantic Regards In Bioscience Text, Proc. 42nd Ann. Conference on Assoc. for Computational Linguistics, 430, 2004.

Seeker L and Cohen K.B, Biomedical Language Processing, What's Beyond Pubmed?, Molecular Cellular, 21 (5), 2006, 589-594. 